\title{
Calculation of the throwing angle of a fertilizer centrifugal device as functions of random coordinates of feeding point
}

\author{
Vasiliy Chernovolov ${ }^{1}$, Lyudmila Kravchenko ${ }^{2, *}$, Alla Nikitina $^{3,4}$, Vladimir Litvinov ${ }^{1,2}$ \\ ${ }^{1}$ Azov-Black Sea Engineering Institute of Don State Agrarian University, 21, Lenina, 347740, \\ Zernograd, Russia \\ ${ }^{2}$ Don State Technical University, Gagarin sq., 1, 344003, Rostov-on-Don, Russia \\ ${ }^{3}$ Supercomputers and Neurocomputers Research Center, 106, lane Italian, 347900, Taganrog, Russia \\ ${ }^{4}$ Southern Federal University, lane Italian, 106, 347900, Taganrog, Russia
}

\begin{abstract}
Liquid fertilizers fed into centrifugal device are spread along angle and radius of feed under action of blades. This article describes how to calculate throwing angular characteristics using Mathcad. The package consists of four programs. Program Mf is intended for calculation of probability density of supply point coordinates under assumption of bivariant normal distribution of system $\mathrm{r}, \gamma$, which are specified in the form of vectors. The result of the calculation is displayed as matrix Mf. The program $\mathrm{M} \alpha$ calculates the throwing angle for all combinations $\mathrm{r}, \gamma$. . To calculate the throwing angle, the method of solving differential equations of particle movement along the blade of the device with input data was used: Radius of the disk R, angular speed $\omega$, coefficient of friction of fertilizers on the blade $\mathrm{f}$. The program Ms extracts from the matrix Mf elements corresponding to a throw angle less than a given number $\mathrm{A}$. The program $F(A)$ sums the elements of the matrix Ms. We obtained the values of the throw angle distribution function by multiplying the resulting sum by the intervals of vectors $r$ and $\gamma$. The calculated throwing angle distribution function is approximated by the standard normal distribution function.
\end{abstract}

\section{Introduction}

Centrifugal devices for distribution of mineral fertilizers are widely used because of their simplicity, high productivity, and easy loading. The theory of the operation of centrifugal devices has been significantly developed $[1,2]$. However, the theory considers the motion of single particles, which limits the use of these dependencies in design calculations. Flowing fertilizers fed to the distributing device get a spread in the angle and radius of the feed under the impact of the shovels. Therefore, programs for calculating the throwing angle should be updated so that the throwing angle is determined as a function of two random arguments - the polar coordinates of the feed points.

\footnotetext{
"Corresponding author: lusya306@yandex.ru
} 
The throwing angle a (Figure 1) relative to the line of motion is determined by the formula

$$
\alpha=\lambda+\omega \cdot t_{1}+\theta-\pi
$$

where $\lambda$ - an angular coordinate of the feed point of the particle, $\theta$ is the angle between the absolute velocity and the radius vector of the particle, $\omega \mathrm{t}_{1}$ is the angle of descent of the particle, i.e. the angular sliding of the particle in absolute motion until it leaves the shovel. The angle of descent of the particle is a function of the radius of the feed from the disk $\mathrm{r}_{0} \omega \mathrm{t}_{1}$ and is defined as the multiplication of the angular velocity of the disk by the time of the sliding of the particle along the shovel.

For a radial shovel, the equation has the form

$$
\omega t=\frac{\cos \varphi}{1-\sin \varphi} \ln \frac{2 R}{r_{0}(1+\sin \varphi)}
$$

We substitute (2) into (1) and get the throwing angle $\alpha$ as a function of random arguments $r_{0}$ and $\lambda$.

$$
\alpha=\lambda+\frac{\cos \varphi}{1-\sin \varphi} \ln \frac{2 R}{r_{0}(1+\sin \varphi)}+\theta-\pi
$$

where the angles $\alpha, \lambda$ are measured from the longitudinal coordinate axis in the direction of rotation of the disk.

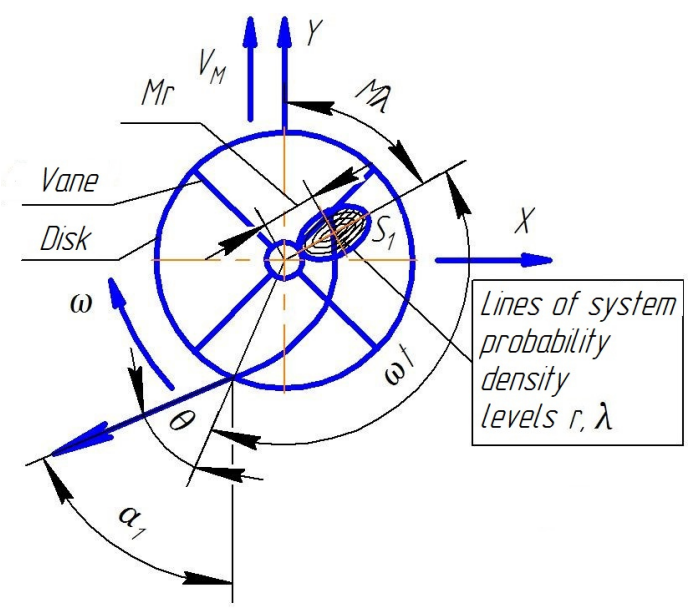

Fig. 1. Determination of the kinematic parameters of the particle when sliding along the shovel.

The given constant value of the throwing angle can be obtained only with a certain combination of arguments satisfying the equation which, after substitution and rearrangement, has the form

$$
r(\alpha)=\frac{2 R}{1+\sin \varphi} \exp \left(-\frac{(1-\sin \varphi)(\alpha-\theta-\lambda)}{\cos \varphi}\right.
$$


The last equation in the polar coordinates $r, \lambda$ gives a logarithmic spiral [1] of the form $\rho=\mathrm{a} \cdot \exp (\kappa \lambda)$, where $\mathrm{a}$ and $\mathrm{k}$ are the parameters of the spiral determined by the dependencies:

$$
\begin{aligned}
& a=\frac{2 R}{1+\sin \varphi} \exp \left(-\frac{1-\sin \varphi}{\cos \varphi}(\alpha-\theta)\right) ; \\
& k=\frac{1-\sin \varphi}{\cos \varphi} .
\end{aligned}
$$

Fertilizer feed to any point of the spiral provides a set constant value of the angle $\alpha$. The shape of the spiral depends only on the angle of friction of the particles on the shovels, while its location depends on $\varphi, \alpha$ and $\mathrm{R}$.

The spiral, built at $\alpha=\alpha 1$, divides the feed zone into two parts (Figure 1) [1]. The combinations $\mathrm{r}_{0}, \lambda$, belonging to the area $\mathrm{S} 1$ located to the right of the spiral, provide an angle $\alpha$ less than $\alpha_{1}$, while at the combinations $\left(r_{0}, \lambda\right)$ not belonging to the area $S 1$, we have $\alpha>\alpha 1$.

The distribution function of a random variable $\alpha$ can be obtained by integrating the density of the system $r_{0}, \lambda$ over the area $S_{1}$.

Let us consider the application of the method for calculating the throwing angle for a device with radial shovels.

\section{Algorithm for calculating}

1. We should record initial data: mathematical expectation of the radius of the feed $\mathrm{Mr}$; standard deviation of the radius of the feed or; mathematical expectation of the angular coordinate of the fertilizer feed point $\mathrm{M} \lambda$; standard deviation of the angular coordinate of the feed $\sigma \lambda$; radius of the distribution disk R; angle of inclination of the shovel to the radius $\psi$; friction coefficient of fertilizers on the shovel $\mathrm{f}$; angular speed of the disk $\omega$.

2. We should create vectors $\mathrm{r}_{\mathrm{i}} ; \lambda_{\mathrm{j}}$ with the number of elements $\mathrm{Nr}=\mathrm{N} \lambda=12$. So the intervals between the elements of the vectors are equal to $0,5 \cdot \sigma r, 0,5 \cdot \sigma \lambda$.

$$
\begin{gathered}
r_{i}=i \cdot 0,5 \cdot \sigma r+M r-3 \cdot \sigma r \\
\lambda_{j}=j \cdot 0,5 \cdot \sigma \lambda+M \lambda-3 \cdot \sigma \lambda
\end{gathered}
$$

3. We should create a probability density matrix for the bivariate distribution of the coordinates of feed $r_{i} ; \lambda_{j}$. We consider random variables to be independent, therefore, the probability density for all combinations is determined by Program Mf for a bivariate normal distribution.

4. We should create a matrix M $\alpha$ of throwing angles for all combinations $r_{i} ; \lambda_{j}$. 


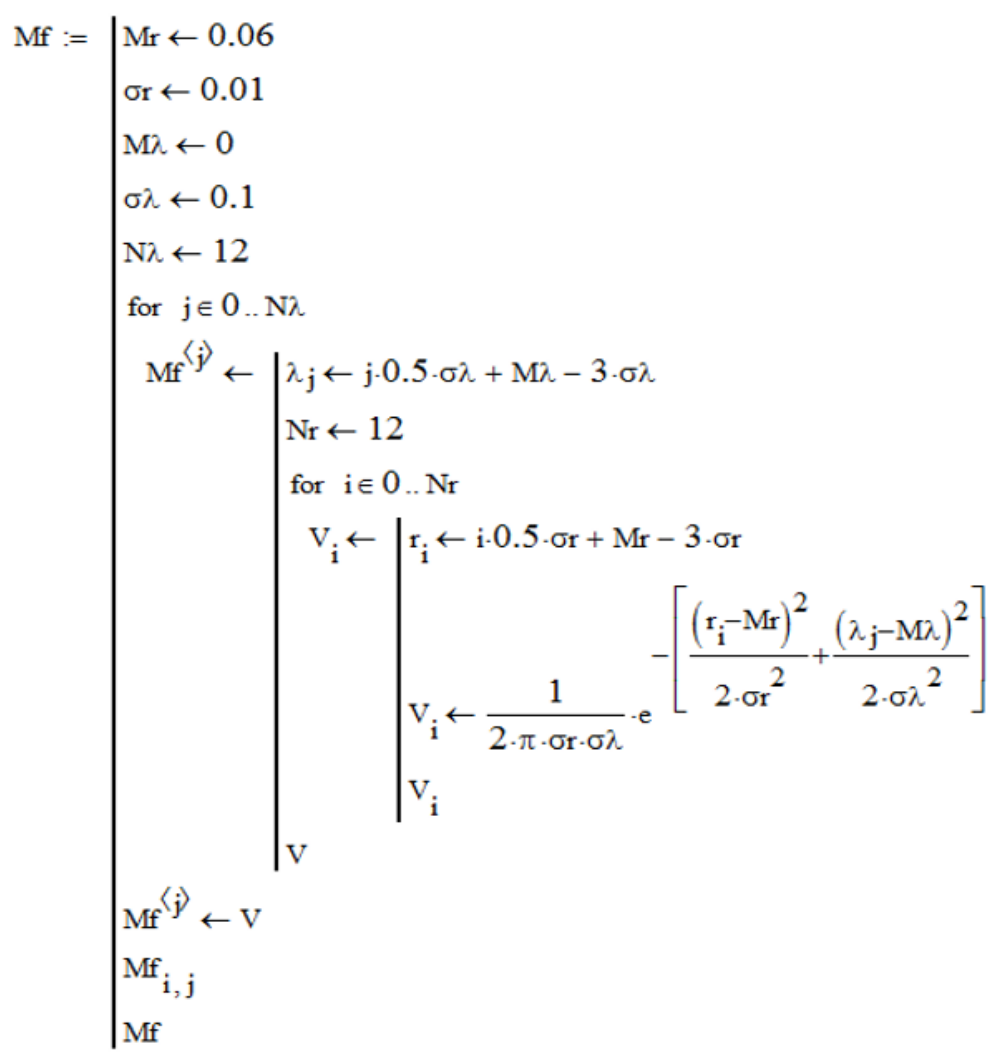

Fig. 2. Program for calculating probability density.

Calculation result is displayed in the form of a matrix (Figure 3)

Mf $=\left(\begin{array}{ccccccccccccc}0.02 & 0.078 & 0.239 & 0.574 & 1.072 & 1.56 & 1.768 & 1.56 & 1.072 & 0.574 & 0.239 & 0.078 & 0.02 \\ 0.078 & 0.307 & 0.946 & 2.27 & 4.241 & 6.171 & 6.993 & 6.171 & 4.241 & 2.27 & 0.946 & 0.307 & 0.078 \\ 0.239 & 0.946 & 2.915 & 6.993 & 13.064 & 19.008 & 21.539 & 19.008 & 13.064 & 6.993 & 2.915 & 0.946 & 0.239 \\ 0.574 & 2.27 & 6.993 & 16.775 & 31.339 & 45.599 & 51.67 & 45.599 & 31.339 & 16.775 & 6.993 & 2.27 & 0.574 \\ 1.072 & 4.241 & 13.064 & 31.339 & 58.55 & 85.19 & 96.532 & 85.19 & 58.55 & 31.339 & 13.064 & 4.241 & 1.072 \\ 1.56 & 6.171 & 19.008 & 45.599 & 85.19 & 123.95 & 140.454 & 123.95 & 85.19 & 45.599 & 19.008 & 6.171 & 1.56 \\ 1.768 & 6.993 & 21.539 & 51.67 & 96.532 & 140.454 & 159.155 & 140.454 & 96.532 & 51.67 & 21.539 & 6.993 & 1.768 \\ 1.56 & 6.171 & 19.008 & 45.599 & 85.19 & 123.95 & 140.454 & 123.95 & 85.19 & 45.599 & 19.008 & 6.171 & 1.56 \\ 1.072 & 4.241 & 13.064 & 31.339 & 58.55 & 85.19 & 96.532 & 85.19 & 58.55 & 31.339 & 13.064 & 4.241 & 1.072 \\ 0.574 & 2.27 & 6.993 & 16.775 & 31.339 & 45.599 & 51.67 & 45.599 & 31.339 & 16.775 & 6.993 & 2.27 & 0.574 \\ 0.239 & 0.946 & 2.915 & 6.993 & 13.064 & 19.008 & 21.539 & 19.008 & 13.064 & 6.993 & 2.915 & 0.946 & 0.239 \\ 0.078 & 0.307 & 0.946 & 2.27 & 4.241 & 6.171 & 6.993 & 6.171 & 4.241 & 2.27 & 0.946 & 0.307 & 0.078 \\ 0.02 & 0.078 & 0.239 & 0.574 & 1.072 & 1.56 & 1.768 & 1.56 & 1.072 & 0.574 & 0.239 & 0.078 & 0.02\end{array}\right)$

Fig. 3. Calculation result of probability density.

Program M $\alpha$ (Figure 4) has two cycles where it changes the polar coordinates of the fertilizer feed points and calculates the throwing angle of the disk with radial shovels as a function of the polar coordinates of the feed points [2]. 


$$
\begin{aligned}
& \mathrm{M} \alpha:=\mid \begin{array}{l}
\mathrm{Mr} \leftarrow 0.06 \\
\sigma r \leftarrow 0.01 \\
\mathrm{M} \lambda \leftarrow-0.4 \\
\sigma \lambda \leftarrow 0.1 \\
\mathrm{~N} \lambda \leftarrow 12 \\
\text { for } \mathrm{j} \in 0 . . \mathrm{N} \lambda \\
\langle j\rangle
\end{array} \\
& \mathrm{M} \alpha^{\langle j\rangle} \leftarrow \mid \begin{array}{l}
\lambda_{\mathrm{j}} \leftarrow \mathrm{j} \cdot 0.5 \cdot \sigma \lambda+\mathrm{M} \lambda-3 \cdot \sigma \lambda \\
\mathrm{Nr}_{\mathrm{r}} \leftarrow 12
\end{array} \\
& \text { for } \mathrm{i} \in 0 . . \mathrm{Nr} \\
& \mathrm{V}_{\mathrm{i}} \leftarrow \mid \mathrm{r}_{\mathrm{i}} \leftarrow \mathrm{i} \cdot 0.5 \cdot \sigma \mathrm{r}+\mathrm{Mr}-3 \cdot \sigma \mathrm{r} \\
& \mathrm{V}_{\mathrm{i}} \leftarrow \mid \mathrm{f} \leftarrow 0.5 \\
& \mathrm{R} \leftarrow 0.3 \\
& \omega \leftarrow 100 \\
& \mathrm{~g} \leftarrow 9.8 \\
& A \leftarrow \frac{f \cdot g}{\omega^{2}} \\
& \phi \leftarrow \operatorname{atan}(f) \\
& \lambda 1 \leftarrow-\mathrm{f} \cdot \omega+\sqrt{(\mathrm{f} \cdot \omega)^{2}+\omega^{2}} \\
& \lambda 2 \leftarrow-f \cdot \omega-\sqrt{(f \cdot \omega)^{2}+\omega^{2}} \\
& B \leftarrow r_{i} \\
& \xi \mathrm{R} \leftarrow \mathrm{R}-\mathrm{r}_{\mathrm{i}} \\
& t \leftarrow 0.01 \\
& \mathrm{t} 1 \leftarrow \operatorname{root}\left[\left[(\mathrm{B}-\mathrm{A}) \cdot\left[\left(\frac{\lambda 2}{\lambda 2-\lambda 1} \cdot \exp (\lambda 1 \cdot \mathrm{t})-\frac{\lambda 1}{\lambda 2-\lambda 1} \cdot \exp (\lambda 2 \cdot \mathrm{t})\right)-1\right]-\xi \mathrm{R}\right], \mathrm{t}\right] \\
& \operatorname{Vr} \leftarrow \frac{\lambda 1 \cdot \lambda 2 \cdot(\mathrm{B}-\mathrm{A})}{\lambda 2-\lambda 1} \cdot(\exp (\lambda 1 \cdot \mathrm{t} 1)-\exp (\lambda 2 \cdot \mathrm{t} 1)) \\
& \Theta \leftarrow \operatorname{atan}\left(\frac{\omega \cdot \mathrm{R}}{\mathrm{V}_{\mathrm{r}}}\right) \\
& A 1_{i} \leftarrow\left(\lambda_{j}+\omega \cdot t 1+\Theta\right)-\pi \\
& \mathrm{v}_{\mathrm{i}} \leftarrow \mathrm{A}_{\mathrm{i}} \\
& \mathrm{v}_{\mathrm{i}} \\
& \mathrm{v}_{\mathrm{i}} \\
& \mathrm{M}^{\langle j\rangle} \leftarrow \mathrm{V} \\
& \mathrm{M} \alpha_{\mathrm{i}, \mathrm{j}} \\
& \mathrm{M} \alpha
\end{aligned}
$$

Fig. 4. Program for calculating the throwing angle of fertilizers with a centrifugal device as a function of two arguments: an angle and a radius of the feed.

Calculation result is displayed in the form of a matrix (Figure 5). 


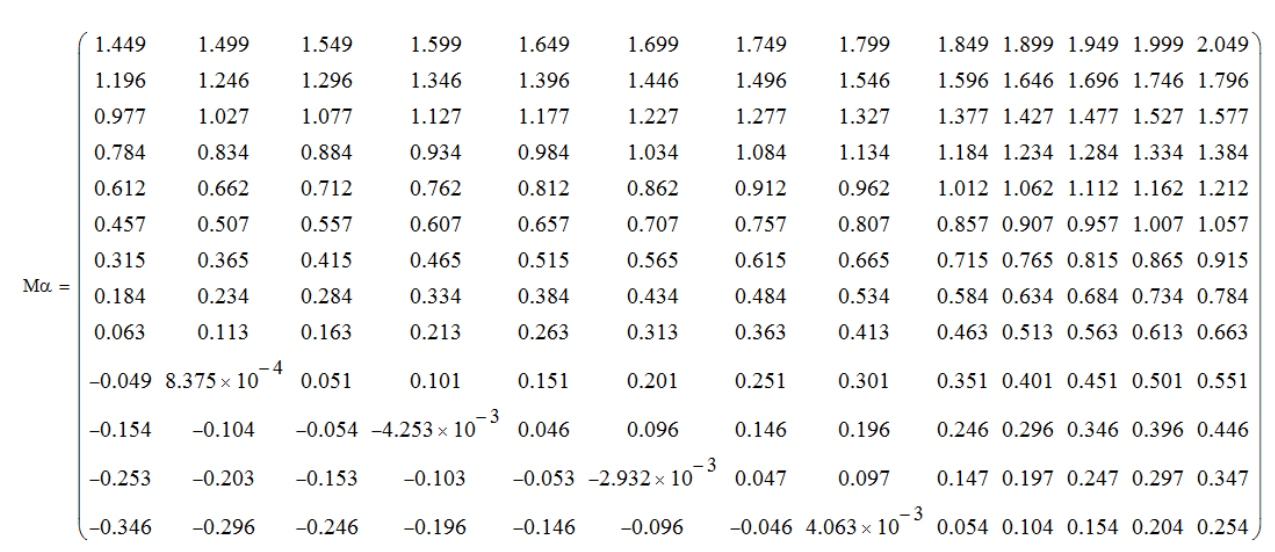

Fig. 5. Display output of the calculation result by Program M $\alpha$.

The matrix $\mathrm{M} \alpha$ can determine the mathematical expectation of the throwing angle. It is necessary for a two-disk device to obtain $=0.6 \mathrm{rad}$, for a one-disk device it has $\mathrm{M} \alpha=0$. These values must be obtained for the center of the matrix. One can correct them by changing $\mathrm{M} \lambda$.

5. One should select from the matrix Mf the elements corresponding to a throwing angle less than that given by number A. By summing the selected matrix elements and multiplying the sum by the intervals of the vectors, we obtain the value of the distribution function of the throwing angle.

The selection of the elements of the matrix Mf is performed by $\operatorname{Program} \operatorname{Ms}(A)$ (Figure 6 ), the result of which is presented in Figure 7.

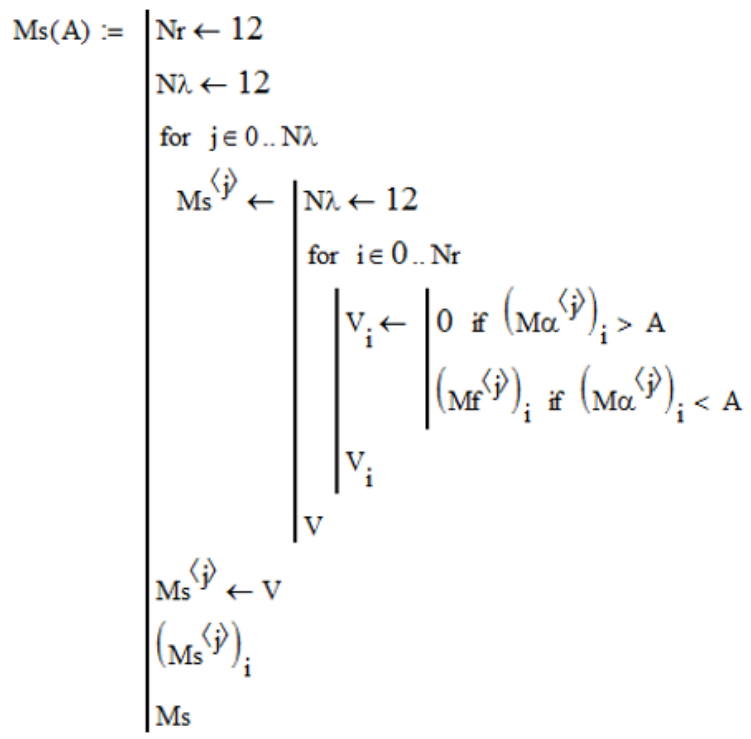

Fig. 6. Program for selecting matrix elements by condition $\mathrm{M} \alpha<\mathrm{A}$. 


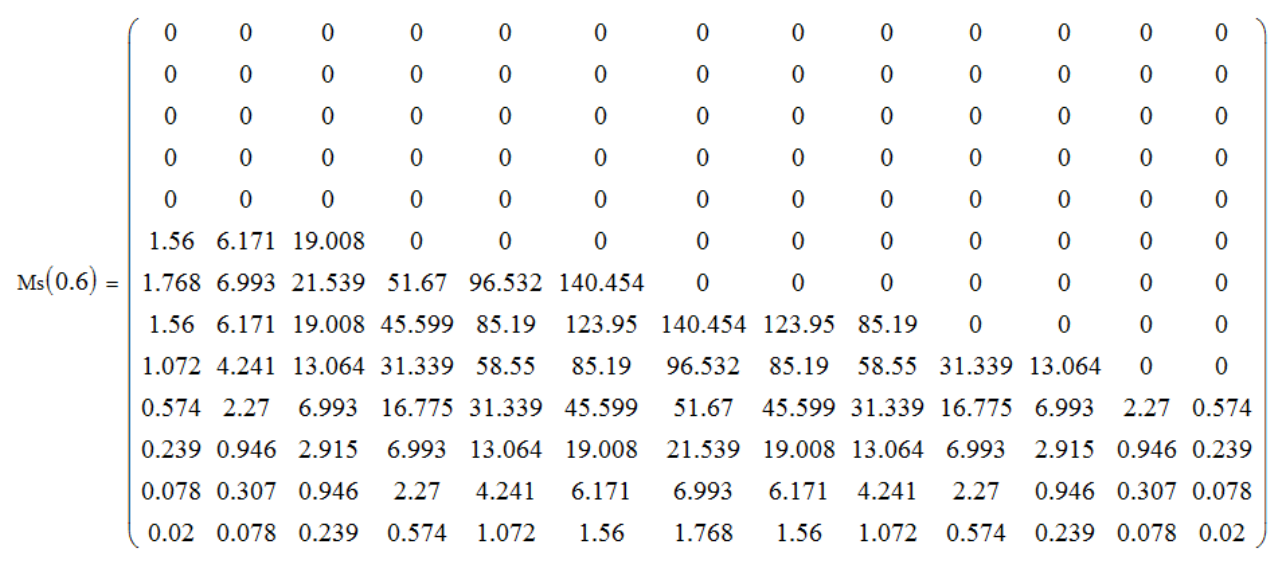

Fig. 7. Matrix for calculating the matrix Ms.

In order to find the values of the distribution function of the throwing angle, we use Program F $(A)$ (Figure 8).

$$
\begin{aligned}
& \mathrm{j}:=0 . .12 \quad \sigma \lambda:=0.3 \quad \sigma \mathrm{r}:=0.01 \\
& \mathrm{~F}(\mathrm{~A}):=\mid \begin{array}{l}
\mathrm{N} \lambda \leftarrow 12 \\
\text { for } \mathrm{j} \in 0 . . \mathrm{N} \lambda \\
\mid \begin{array}{l}
\mathrm{v}_{\mathrm{j}} \leftarrow \sum \mathrm{Ms}(\mathrm{A}) \\
\mathrm{v}_{\mathrm{j}}
\end{array} \\
\mathrm{V}\rangle \mathrm{j}\rangle \\
\mathrm{F} 1 \leftarrow \sum \mathrm{V} \\
\mathrm{F} \leftarrow \mathrm{F} 1 \cdot 0.5 \cdot \sigma \mathrm{r} \cdot 0.5 \cdot \sigma \lambda
\end{array}
\end{aligned}
$$

Fig. 8. Program for calculating the values of the distribution function of the throwing angle.

The approximation of the obtained distribution function of the throwing angle by the standard normal distribution function (Figure 9) turns out to be in a good agreement with the results. The mathematical expectation of the throwing angle, obtained by calculation, corresponds to the optimal value for a two-disk device. The standard deviation of the throwing angle is less than the optimum one being equal to 0.6 radians. It can be increased in two ways: by spreading the feed along the angle $\lambda$ or by applying the disk shovels deflected forward to the direction of rotation. In the latter case, one should replace Program $\mathrm{M} \alpha[1,3]$ with Program Disk- $\psi$. 


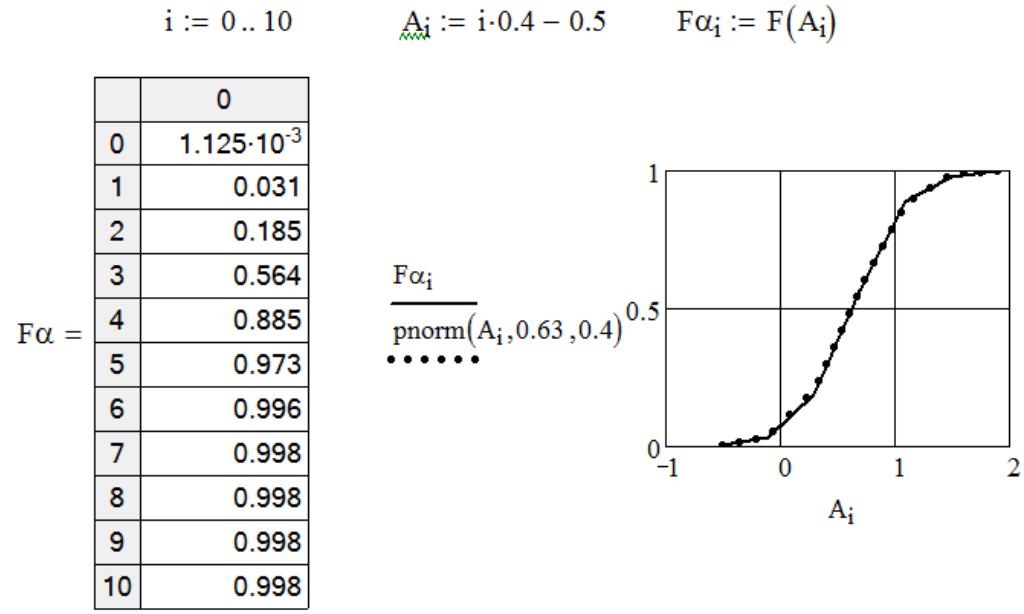

Fig. 9. Calculation result for the distribution function of the throwing angle.

\section{Conclusions}

1. The method for calculating the distribution function of the throwing angle of fertilizers by a centrifugal device as a function of the random coordinates of feed points was verified by a numerical illustration.

2. The distribution function of the throwing angle obtained by calculation is approximated by the standard normal distribution function. The mathematical expectation of the throwing angle is obtained as close to the optimal value for the two-disk device. The standard deviation of the throwing angle obtained by calculation is less than the optimal value. Therefore, it should be recommended to repeat the calculation with the shovels deflected forward to the direction of rotation.

3. An increase in the standard deviation of the throwing angle can be obtained by spreading the feed of fertilizers to the disk, for example, by applying feed through two fertilizer guides. In this case, the probability density of the system $r, \lambda$ should be calculated by the superposition formula of bivariate normal distributions.

\section{References}

1. P.K. Agrawal, S. Agrawal, R. Shrivastava, Biotech 5, 853-866 (2015)

2. S.L. Berglund, G.I. Ågren, Oikos 121, 1112-1120 (2012)

3. B.L. Bodirsky, A.Popp, H. Lotze-Campen, J.P. Dietrich, S. Rolinski, I. Weindl, C. Schmitz, C. Müller, M. Bonsch, F. Humpenöder, Nat. Commun 5 (2014)

4. A. Zafari, M. Kianmehr, Environ. Technol. 35, 478-486 (2014)

5. A.M.F. Da Silva, N. Boukhdoud, R.Gros, Geoderma 269, 1-9 (2016)

6. B. Gu, X. Ju, J. Chang, Y. Ge, P.M. Vitousek, Proc. Natl. Acad. Sci. U.S.A. 112 (2015)

7. E. Ikenganyia, U. Ndubuaku, C. Onyeonagu, U. Ukonze, Am. J. Exp. Agric. 6, 147 (2015)

8. Y. Kuzyakov, E. Blagodatskaya, Soil Biol. Biochem. 83, 184-199 (2015)

9. T.D. Loecke, C.A. Cambardella, M. Liebman, Nutr. Cycl. Agroecosyst. 93, 65-74 (2012) 
10. Q. Meng, W. Yang, M. Men, A. Bello, X. Xu, B. Xu, L. Deng, X. Jiang, S. Sheng, X. $\mathrm{Wu}$, Front. Microbiol 10, 529 (2019)

11. J.M. Norton, J.P. Schimel, Handbook of Soil Science, 8-18 (2011)

12. M. Redin, S. Recous, C. Aita, G. Dietrich, A.C. Skolaude, W.H Ludke, R. Schmatz, S.J. Giacomini, Soil Biol. Biochem. 78, 65-75 (2014)

13. J.P. Reganold, J.M. Wachter, Nat. Plants 2, 15221 (2016)

14. M.S. Reiter, T.C. Daniel, N.A. Slaton, R.J. Norman, Soil Sci. Soc. Am. J. 78, 861 (2014)

15. A.A.S. Sinegani, J. Maghsoudi, The effects of water potential on some microbial populations and decrease kinetic of organic carbon in soil treated with cow manure under laboratory conditions (2011)

16. V.A. Chernovolov, V.A. Kravchenko, L.V. Kravchenko, A.Yu. Nesmiyan, V.I. Khizhnyak, S.A. Sherstov, Amazonia Investiga (2018) 